\title{
cis-Element Combinations Determine Phenylalanine Ammonia-Lyase Gene Tissue-Specific Expression Patterns
}

\author{
Antonio Leyva, ${ }^{a, 1}$ Xiaowu Liang, ${ }^{a, b, 2}$ José A. Pintor-Toro, ${ }^{a, 3}$ Richard A. Dixon, ${ }^{c}$ \\ and Christopher J. Lamba,4 \\ a Plant Biology Laboratory, Salk Institute for Biological Studies, 10010 North Torrey Pines Road, La Jolla, California 92037 \\ b Graduate Program in Biology, University of California at San Diego, La Jolla, California 92093 \\ c Plant Biology Division, Samuel Roberts Noble Foundation, P.O. Box 2180, Ardmore, Oklahoma 73402
}

The bean phenylalanine ammonia-lyase gene 2 (PAL2) is expressed in the early stages of vascular development at the inception of xylem differentiation, associated with the synthesis of lignin precursors. This is part of a complex program of developmental expression regulating the synthesis of functionally diverse phenylpropanoid natural products. Analysis of the expression of PAL2 promoter- $\beta$-glucuronidase gene fusions in transgenic tobacco plants showed that functionally redundant cis elements located between nucleotides -289 and -74 relative to the transcription start site were essential for xylem expression, but were not involved in expression in leaf primordia and stem nodes or in establishing tissue specificity in petals. The -135 to -119 region implicated in xylem expression contains a negative element that suppresses the activity of a cryptic cis element for phloem expression located between -480 and -289 . The functional properties of each vascular element are conserved in stem, petiole, and root, even though the xylem and phloem are organized in different patterns in these organs. We conclude that the PAL2 promoter has a modular organization and that tissue-specific expression in the vascular system involves a negative combinatorial interaction, modulation of which may provide a flexible mechanism for modification of tissue specificity.

\section{INTRODUCTION}

Phenylalanine ammonia-lyase (PAL; EC 4.3.1.5) catalyzes the first reaction in the synthesis from phenylalanine of a wide range of natural products based on the phenylpropane skeleton, including cinnamyl alcohols, which are the substrates for the peroxidase-mediated oxidative reactions that generate the lignin polymer (Lamb et al., 1989). Dissection of stems reveals high levels of PAL enzyme activity specifically in vascular tissues (Rubery and Northcote, 1968), and the induction of xylem differentiation in callus culture by manipulation of auxin levels is accompanied by a marked increase in PAL activity (Haddon and Northcote, 1975). These data suggest that PAL is a key regulatory step in lignin synthesis during xylogenesis, and this has recently been confirmed by gene transfer experiments in which a reduction of PAL activity in transgenic tobacco plants, engineered by introduction of heterologous PAL

\footnotetext{
${ }^{1}$ Current address: Instituto Nacional de Investigaciones Agrarias, Madrid, Spain.

2 Current address: USDA-Plant Gene Expression Center, 800 Buchanan Street, Albany, CA 94710.

${ }^{3}$ Current address: Instituto Recursos Naturales y Agrobiologia, Sevilla, Spain.

${ }^{4}$ To whom correspondence should be addressed.
}

sequences, resulted in a marked reduction in the lignification of tracheary element cell walls (Elkind et al., 1990).

PAL is encoded by a small family of differentially regulated genes (Cramer et al., 1989; Lois et al., 1989; Minami et al., 1989; Ohl et al., 1990), leading to the selective synthesis of functional variants of the enzyme (Bolwell et al., 1985; Liang et al., 1989a). In bean, the transcript encoded by the PAL2 gene accumulates to high levels in roots and stems (Liang et al., 1989a), suggesting a major role for PAL2 in the synthesis of lignin monomers. Moreover, transgenic tobacco plants containing the reporter gene $\beta$-glucuronidase (GUS) under the control of the PAL2 promoter show strong GUS staining in vascular tissues, specifically in differentiating xylem cells (Bevan et al., 1989; Liang et al., 1989b). Within this cell lineage GUS staining can first be observed prior to the appearance of $x y$ lem morphological features in juvenile tissues immediately adjacent to the shoot apical meristem (Liang et al., 1989b). These observations indicate that transcriptional activation of the PAL2 gene is an early event in vascular development at the inception of xylem differentiation. Thus, the PAL2 promoter responds to a signal pathway that is activated by cues for vascularization, and delineation of the cis elements involved in vascular expression would provide the basis for biochemical and genetic dissection of this morphogenetic pathway. 
Vascular expression is part of a complex program of PAL2 promoter activity (Bevan et al., 1989; Liang et al., 1989b). PAL2 is induced in epidermal tissues and perivascular parenchyma in response to wounding or irradiation associated with the synthesis of protective flavonoids, and is also expressed in petals associated with the synthesis of anthocyanin pigments. In addition, strong GUS staining is observed in stem nodes, as well as in root and shoot apices, where PAL2 may be involved in the synthesis of growth-promoting dehydrodiconiferyl glucosides that mediate cytokinin action (Lynn and Chang, 1990).

In this article, we present the effects of mutations in the PAL2 promoter on the expression of GUS gene fusions in transgenic tobacco plants to identify regions involved in vascular expression and to evaluate their organization within the overall functional architecture of this complex promoter. We show that the region from -289 to -74 contains redundant cis elements for xylem expression and a negative element that suppresses the activity of upstream elements for expression in phloem and perivascular parenchyma. Deletion of the xylem elements does not affect the tissue specificity of the promoter in petals or expression in leaf primordia and nodes. These data indicate that the PAL2 promoter has a modular organization and that tissue specificity in the vascular system is established by a novel negative combinatorial interaction between cis elements.

\section{RESULTS}

Xylem cis Elements in the Proximal Region of the Promoter

The sequence of the PAL2 gene has been described by Cramer et al. (1989), and the functional properties of the promoter were examined by analysis of GUS activity in transgenic tobacco plants containing PAL2 sequences from -1173 to +158 (relative to the transcription start site) translationally fused to the GUS reporter gene (Liang et al., 1989b). The full-length promoter to -1173 gives high levels of PAL2-GUS expression in transgenic tobacco stems (Liang et al., 1989b), and Figure 1 shows that 5 ' deletions to -613 and -444 progressively reduced the level of extractable GUS activity in stems. If the promoter was further deleted to -289 or beyond, no significant GUS activity above background levels in plants transformed with a promoterless GUS construct was observed (Figure 1). Histochemical analysis of GUS activity in situ in stem lateral cross-sections demonstrated that deletions up to and including $\mathbf{- 4 4 4}$ gave the same pattern as that observed for the full-length promoter as shown in Figure $2 \mathrm{~A}$, with staining specifically localized to differentiating xylem in the inner region of the vascular cylinder. No GUS staining was observed in the internal phloem bundles located in the central pith tissue or in vascular tissues external to the xylem including the external phloem and perivascular parenchyma. Figure 3 depicts the organization of phloem and xylem tissues in tobacco stems, petioles, and roots.

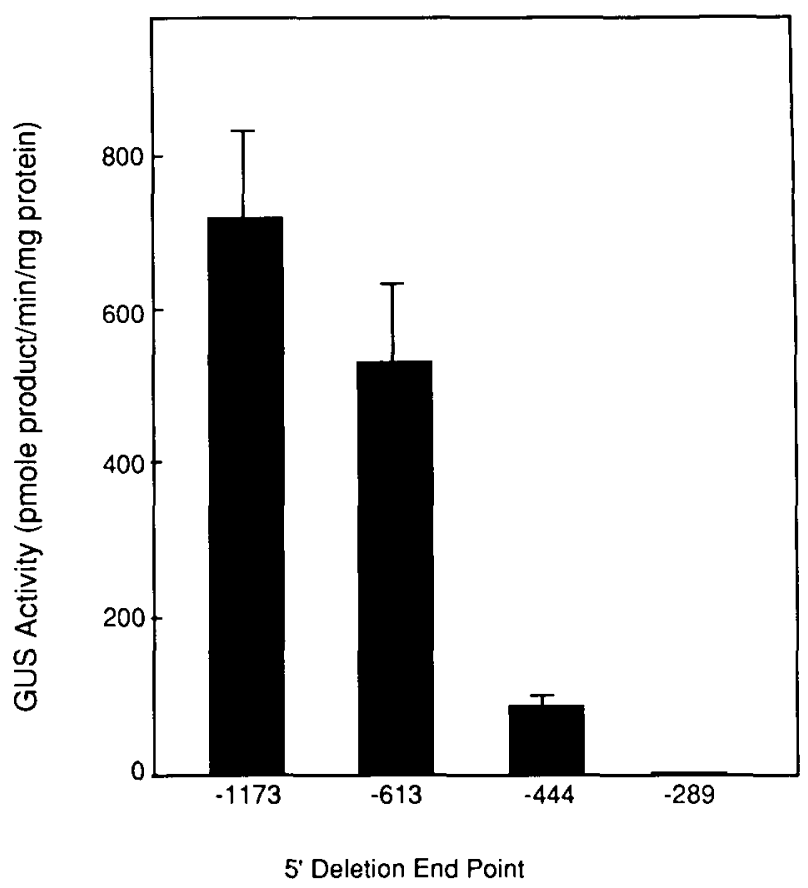

Figure 1. Effect of 5' Deletions on PAL2-GUS Expression in Stems.

GUS activity was measured in extracts of stem tissue between the second to fourth nodes from $8-$ to $10-\mathrm{cm}$ tall explants of primary transformants. Data are presented as the mean and SD of GUS activity in independent transformants containing the same construct. The mean GUS activity in stem extracts from transformants containing the -289 deletion construct was $6 \mathrm{pmol} / \mathrm{min} / \mathrm{mg}$ protein compared with a GUS activity of $11 \mathrm{pmol} / \mathrm{min} / \mathrm{mg}$ protein in equivalent extracts from a plant transformed with a promoterless GUS construct as a negative control.

\section{Multiple cis Elements for Vascular Expression}

We next located cis elements within the proximal region that are involved in establishing tissue specificity by analysis of a series of internal deletions in the context of the full-length promoter. As in the case of the $5^{\prime}$ deletions, the internal deletions contained PAL2 sequences to +158 translationally fused to GUS. Internal deletion from -119 to $-74(\Delta 119-74)$ resulted in the same spatial pattern of expression in the stem vascular system as that observed with the intact promoter (Figure 2D). However, deletion from -135 to -74 ( $\Delta$ 135-74) led to a new pattern of expression. Thus, although some GUS staining in the rays of xylem parenchyma characteristic of the full promoter could still be observed, there was now strong GUS staining in the internal phloem, the external phloem, and surrounding perivascular parenchymal tissue (Figures 2D and $2 \mathrm{G})$. Further deletion from -289 to -74 ( $\Delta$ 289-74) abolished expression in xylem, but strong expression in phloem tissues was retained (Figures $2 \mathrm{D}$ and $2 \mathrm{~J}$ ). Finally, deletion from -480 
to $-74(\Delta 480-74)$ caused the loss of all expression in vascular tissues (Figures $2 \mathrm{M}$ and $2 \mathrm{~N}$ ).

The distinctive patterns of PAL2-GUS vascular expression in this set of nested deletions were, with two minor exceptions, highly reproducible among independent transformants containing the same construct, as shown in Table 1. For each construct, tissue specificities were first examined in primary transformants and confirmed by analysis of the progeny. In the case of construct $\Delta 135-74$, seven of 11 transformants exhibited relatively weak GUS staining in xylem compared with phloem and perivascular parenchyma (Figure 2D), whereas the other four transformants showed an intensity of staining in xylem similar to that in these other tissues. With one of the $10 \Delta$ 289-74 transformants, GUS staining was observed only in the internal phloem, whereas the other nine transformants exhibited staining in both internal and external phloem. In some stem sections of $\Delta 119-74, \Delta 135-74$, and $\Delta$ 289-74 transformants, weak, variable staining was observed in the epidermis (e.g., see Figure 2D). Inconsistent epidermal staining was also observed in plants containing the full-length promoter construct and can be ascribed to a weak wound response during the preparation of tissue sections (Liang et al., 1989b). The copy number of PAL2-GUS transgenes in the transformants containing internal deletions varied from one to four, but the copy number did not affect the characteristic patterns of tissue specificity observed with each construct.

These data demonstrated that the -289 to -74 region of the PAL2 promoter contains sequences essential for xylem expression and that there are cryptic cis elements between -480 and -289 for expression in phloem and perivascular parenchyma. Within the xylem region, deletion from -135 to -74 was not sufficient to abolish xylem expression completely, suggesting that sequences important or essential for xylem expression might be located between -289 and -135 . However, transgenic plants containing the construct $\Delta 289-139$, in which sequences between -289 and -139 were deleted, still exhibited strong expression in xylem tissue (Table 1). These observations indicate the presence of redundant cis elements within the -289 to -74 xylem region, although the sequences upstream of -139 and downstream of -135 are not functionally identical, because with $\Delta$ 289-139 GUS staining was restricted to xylem tissues, whereas with $\Delta$ 135-74 (deletion of -135 to -74 ) staining was also observed in phloem and perivascular parenchyma tissues (Table 1, Figures 2D and 2G).

Expression in phloem and perivascular parenchyma only became apparent following deletion of sequences between -135 and -119 ( $\Delta 135-74$ compared with $\Delta 119-74)$, suggesting that this region contains a negative element that inhibits the activity of the upstream $(-480$ to -289$)$ cis elements for expression in these other vascular tissues. The presence of a negative element in this region was confirmed by quantitation of stem GUS activity. The mean GUS activity in stem extracts of $\Delta 135-74$ transformants was $5094 \mathrm{pmol} / \mathrm{min} / \mathrm{mg}$ protein, compared to 2233 and $847 \mathrm{pmol} / \mathrm{min} / \mathrm{mg}$ protein in $\Delta$ 119-74 transformants and plants containing the full-length promoter construct, respectively.
To investigate whether there was a reciprocal interaction between the upstream phloem element and the downstream negative element, we examined the pattern of GUS staining in transgenic plants containing construct $\Delta \mathbf{4 8 0 - 2 8 4}$ in which sequences between -480 and -284 were deleted (Table 1 ). This construct established a xylem-specific pattern of expression indistinguishable from that observed with the full-length promoter. Hence, sequences in this region do not contribute to, or modify, the tissue specificity of the downstream sequences implicated in xylem expression, although the $5^{\prime}$ deletion experiments indicated that the -444 to -289 region enhances the level of expression within the tissue-specific pattern established by the downstream xylem elements.

\section{Conservation of cis Element Functions in Other Organs}

Xylem and phloem tissues are organized differently in the vascular systems of leaf petioles and roots compared with stems (Figure 3), so we therefore examined whether the tissue specificities exhibited by the PAL2 promoter deletion mutants were conserved in these other organs. In petioles, the xylem is arranged in a crescent with two arrays of phloem tissue in arcs above and below the xylem. In roots, the xylem extends in ridgelike projections from the central core of the vascular cylinder toward the surrounding specialized perivascular parenchyma or pericycle. Phloem tissue is located between the ridges of xylem tissue.

The full-length promoter and all deletion mutants that had xylem expression in stems also showed strong GUS staining in the upper sector of the xylem region in petiole lateral crosssections (Figures $2 \mathrm{~B}$ and $2 \mathrm{E}$ ) and at the poles of the ridges where xylem differentiation is initiated in roots (Figures $2 \mathrm{C}$ and $2 F$ ). Likewise, the tissue specificity of construct $\Delta 289-74$ was conserved with GUS staining in petioles, specifically in the two arcs of phloem tissues as well as in the perivascular parenchyma immediately surrounding the lower arc (Figure 2K), and in roots, specifically in the outer layers of phloem tissue between the xylem poles (Figure 2L). Transgenic plants containing this construct showed no xylem staining in either roots or petioles. As in stems, construct $\Delta 135-74$ showed patterns of expression in petioles and roots that were intermediate between those observed with the full-length promoter and construct $\Delta$ 289-74. Thus, in petioles there was weak staining in xylem tissue together with staining in phloem and perivascular parenchyma (Figure $2 \mathrm{H}$ ). In roots, staining at the xylem poles was accompanied by strong staining in the pericycle and surrounding endodermal cells, with weak staining in phloem (Figure 2l).

\section{Function of Vascular cis Elements in Other Modes of PAL2 Expression}

Expression in differentiating xylem is only one aspect of a complex program of PAL2 promoter activity. Although detailed 

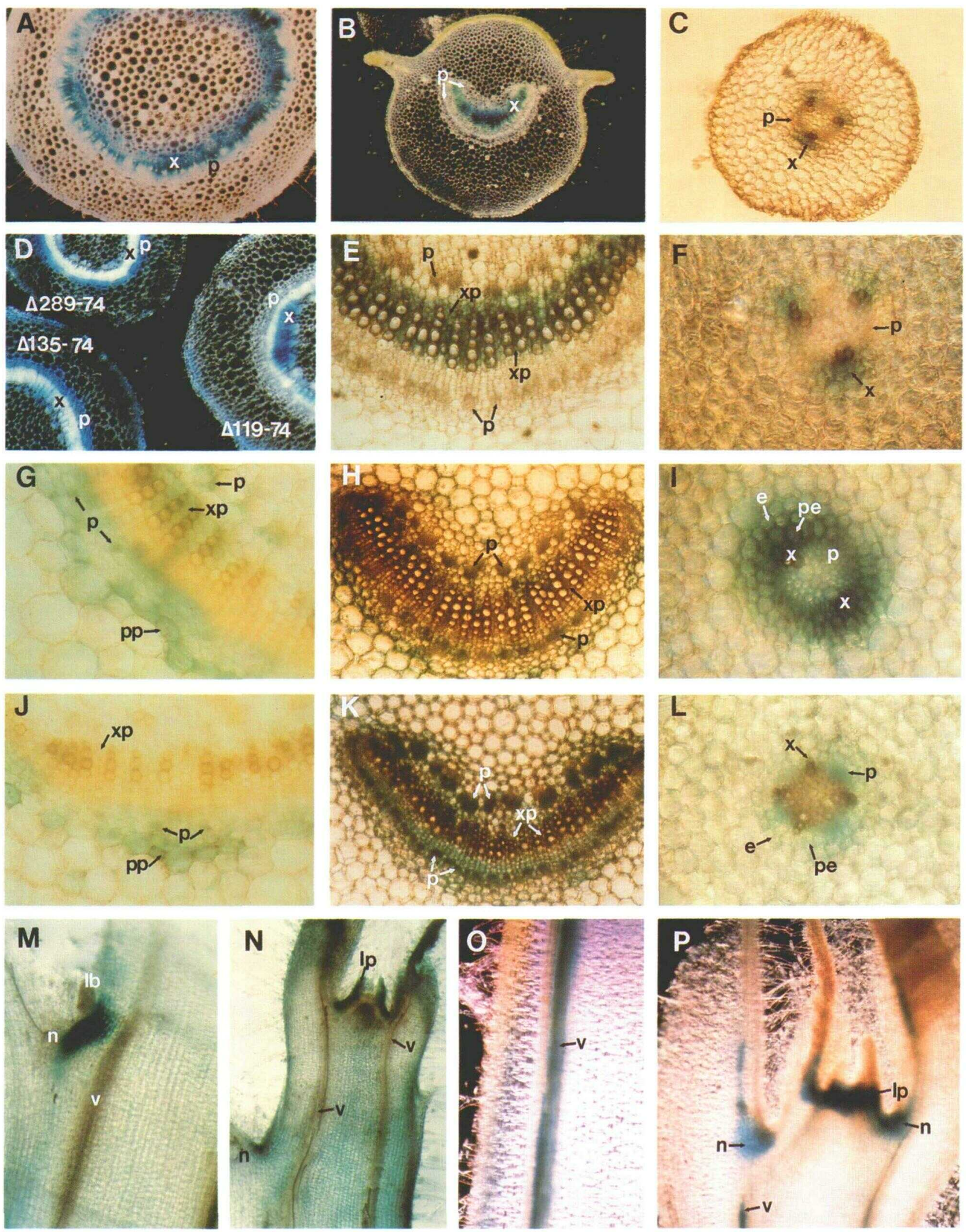

Figure 2. Spatial Pattern of PAL2-GUS Expression in Transgenic Tobacco Plants Revealed by in Situ Staining of GUS Activity. 


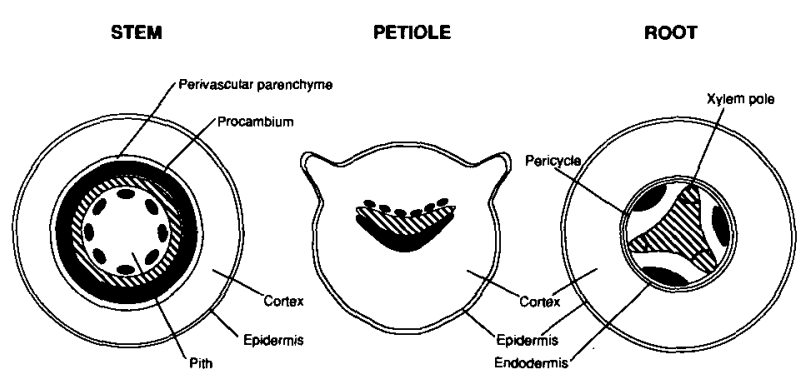

Figure 3. Organization of Phloem and Xylem Tissues in Tobacco Stem, Petiole, and Root.

Modified from Raven et al. (1981). The root cross-section depicts a triarch arrangement. The number of poles can vary in roots of the same plant, and, in tobacco, there can be between two and five. The roots exhibit triarch arrangements in Figures $2 \mathrm{C}$ and $2 \mathrm{~F}$, a diarch in Figure $2 \mathrm{l}$, and a tetrarch in Figure 2L. Black areas show the phloem; the hatched areas indicate the xylem.

dissection of cis elements involved in these diverse modes of expression falls outside the scope of this article, we examined whether internal deletions that affected vascular expression had any impact on the other key aspects of the developmental regulation of the promoter. Figure $2 \mathrm{M}$ shows a longitudinal section of the stem of a transgenic plant containing construct $\Delta 480-74$, with strong GUS staining of nodal tissue underlying the axillary bud in the absence of GUS staining of vascular tissues (compare Figure 2O). Likewise, Figure 2N shows GUS staining in leaf primordia at the shoot apex and in adjacent immature nodes. Unlike plants containing GUS under the control of the full-length PAL2 promoter, $\Delta$ 480-74 transformants showed no GUS staining of the developing leaf vascular traces or the stem vascular system at the shoot apex (compare panels $\mathrm{N}$ and $\mathrm{P}$ in Figure 2). Thus, expression in the shoot apex and in stem nodes was not modulated by the sequences from -480 to -74 that are essential for vascular expression.

In contrast, deletion of this region almost completely abolished expression in petals, as shown in Table 2. However, more limited deletions in this region modulated petal expression differently from vascular expression. Thus, deletion from -135 to -119 ( $\Delta$ 135-74 compared with $\Delta 119-74)$, which leads to expression in phloem and perivascular parenchyma as well as xylem, did not reduce either the overall level of GUS activity in petals or the preferential expression in anthocyanin-accumulating tissue compared to nonpigmented tissue. Deletion from -289 to -74 ( $\Delta$ 289-74), which completely switches expression in the vascular system from xylem to phloem and perivascular parenchyma, markedly reduced the level of GUS activity but did not affect tissue specificity in petals.

\section{DISCUSSION}

Analysis of the pattern of expression of PAL2-GUS gene fusions containing a series of internal deletions in the promoter showed that nucleotide sequences between -289 and -74 were essential for expression in differentiating xylem. However, internal deletion of the sequences between either -289 and -135 , or -135 and -74 separately did not abolish expression in xylem tissue, indicating the presence of partially redundant cis elements. Deletion of the cis elements required for xylem expression had no effect on the activity of the promoter in leaf primordia and stem nodes, implying that the corresponding cis elements are located elsewhere in the promoter and can function independently. Likewise, deletion from -135 to -74 did not affect either the level of GUS activity in petals or the preferential expression in pigmented tissues, and

Figure 2. (continued).

(A) Full-length promoter; stem section ( $\times 30)$.

(B) Full-length promoter; petiole section $(\times 40)$.

(C) Full-length promoter; root section ( $\times 75)$.

(D) $\Delta 119-74, \Delta 135-74$, and $\Delta$ 289-74; stem sections ( $\times 25)$.

(E) Full-length promoter; petiole section $(\times 110)$

(F) Full-length promoter; root section $(\times 150)$.

(G) $\Delta$ 135-74; vascular tissues in stem section $(\times 150)$.

(H) $\Delta$ 135-74; vascular tissues in petiole section $(\times 110)$.

(I) $\Delta$ 135-74; vascular tissues in root section ( $\times 150)$.

(J) $\Delta$ 289-74; vascular tissues in stem section $(\times 150)$.

(K) $\Delta$ 289-74; vascular tissues in petiole section $(\times 110)$.

(L) $\Delta$ 289-74; vascular tissues in root section ( $\times 150)$.

(M) $\Delta 480-74$; longitudinal section through stem node $(\times 35)$.

(N) $\Delta 480-74$; longitudinal section through shoot apex ( $\times 35)$.

(O) Full-length promoter; longitudinal section through stem internode $(\times 50)$.

(P) Full-length promoter; longitudinal section through shoot apex $(\times 50)$.

e, endodermis; lb, lateral bud; lp, leaf primordium; $n$, node; $p$, phloem; pe, pericycle; pp, perivascular (phloem) parenchyma; $v$, vascular tissue; $x$, xylem, xp, xylem parenchyma. 
Table 1. Expression of PAL2 Promoter Internal Deletions in Stem Vascular Tissues

\begin{tabular}{|c|c|c|c|}
\hline Deletion & $\begin{array}{l}\text { Number of } \\
\text { Transformants }\end{array}$ & Vascular Expression Pattern & Variation among Transformants \\
\hline Full-length promoter & 7 & Xylem & - \\
\hline$\Delta 119-74$ & 6 & Xylem & - \\
\hline$\Delta 135-74$ & 11 & $\begin{array}{l}\text { Phloem, perivascular parenchyma, } \\
\text { and xylem }\end{array}$ & $\begin{array}{l}\text { Four show comparable staining } \\
\text { intensities in xylem and } \\
\text { phloem; seven show weak } \\
\text { staining in xylem compared } \\
\text { with phloem. }\end{array}$ \\
\hline$\Delta 289-74$ & 10 & $\begin{array}{l}\text { Phloem and perivascular } \\
\text { parenchyma }\end{array}$ & $\begin{array}{l}\text { One shows staining in internal } \\
\text { phloem but not external } \\
\text { phloem. }\end{array}$ \\
\hline$\Delta 480-74$ & 5 & No vascular expression & - \\
\hline$\Delta 289-139$ & 5 & Xylem & - \\
\hline$\Delta 480-284$ & 8 & Xylem & - \\
\hline
\end{tabular}

-, No variation among transformants.

although deletion from -289 to -74 considerably reduced the level of expression in petals, tissue specificity was retained. These data indicate that the PAL2 promoter has a modular organization, with distinct sets of cis elements involved in different modes of expression.

A particularly striking feature of the functional architecture of the PAL2 promoter was the presence of a cryptic cis element for phloem expression located between -480 and -289 , which became active following removal of sequences between -135 and -119 . This activation is unlikely to result from a change in the spacing of the phloem element relative to TATA proximal elements because deletion from -119 to -74 did not activate the element, whereas a further small deletion to -135 produced a profound effect, even though the phloem cis element remained over $140 \mathrm{bp}$ from the TATA proximal sequences. Likewise, each internal deletion was constructed in the same manner, leading to the introduction of the sequence CGACGTCG, and hence the specific effects of the -135 to -74 deletion cannot be accounted for by the introduction of this sequence. Therefore, we conclude that the activity of the phloem cis element was suppressed by the intrinsic properties of a negative regulatory cis element located between -135 and -119 . This element lies within a region of the PAL2 promoter implicated in xylem expression. Moreover, deletion of these specific sequences not only activated the upstream phloem element, but also appeared to reduce the strength of promoter activity in xylem (Table 1 and Figure 2D, $\Delta$ 135-74 compared with $\Delta 119-74$ ), suggesting multiple functions for this small region of the promoter. Several examples have been reported of cis-acting regulatory sequences that can either activate or repress depending on the cellular context, e.g., a 25-bp sequence of the proliferin promoter confers either positive or negative hormonal regulation depending on cell type (Miesfeld et al., 1987; Sakai et al., 1988).

The dual function of the -135 to -119 region might reflect the presence of more than one independent element within this sequence or overlapping interactive elements. However, our observations best fit the hypothesis that this small region contains a single cis element, which binds a trans-acting factor(s) with multiple regulatory functions. Thus, the sequence

Table 2. PAL2-GUS Expression in Petals

\begin{tabular}{|c|c|c|c|c|}
\hline \multirow[b]{3}{*}{ Construct } & \multirow{3}{*}{$\begin{array}{l}\text { Number of } \\
\text { Transformants }\end{array}$} & \multicolumn{3}{|l|}{ GUS Activity } \\
\hline & & Anthocyanin-Accumulating Tissue & Nonpigmented Tissue & \multirow[b]{2}{*}{ Ratio } \\
\hline & & \multicolumn{2}{|c|}{$(\mathrm{pmol} / \mathrm{min} / \mathrm{mg}$ protein $)$} & \\
\hline Full-length promoter & 5 & $22182 \pm 6310$ & $4776 \pm 611$ & 4.6 \\
\hline$\Delta 119-74$ & 6 & $29927 \pm 9419$ & $4450 \pm 1160$ & 6.7 \\
\hline$\Delta 135-74$ & 5 & $40579 \pm 6855$ & $5581 \pm 1376$ & 7.2 \\
\hline$\Delta 289-74$ & 4 & $2191 \pm 595$ & $505 \pm 173$ & 4.3 \\
\hline$\Delta 480-74$ & 2 & 109 & 116 & 0.9 \\
\hline
\end{tabular}

Data are presented as the mean \pm SE of GUS activities in equivalent tissue extracts from independent transformants. 
-134 TCCACCAACCCC $_{-123}$ in the PAL2 promoter corresponds to a conserved motif found in a number of PAL promoters from bean, parsley, and Arabidopsis, with a consensus sequence of $\mathrm{CCA}(\mathrm{A} / \mathrm{C}) \mathrm{C}(\mathrm{A} / \mathrm{T}) \mathrm{AA} \mathrm{C}(\mathrm{C} / \mathrm{T}) \mathrm{CC}$, which has also been found in a number of other stress-inducible promoters (Lois et al., 1989; Ohl et al., 1990). Inducible in vivo footprints mapping to this sequence in a parsley PAL promoter have been observed associated with transcriptional activation following treatment of cell suspension cultures with UV light or fungal elicitor (Lois et al., 1989). Thus, this specific cis element and the cognate trans-acting factor(s) may be involved in various modes of expression, both during development and also in response to environmental stimuli, and hence may function in the integration of promoter activity.

The generation of new patterns of tissue specificity by combinatorial interactions between cis elements has been reported for the promoter of the cauliflower mosaic virus $35 \mathrm{~S}$ transcript (Benfey and Chua, 1990), and our data have revealed such interactions in a plant cellular promoter. However, whereas the interactions between cis elements in the cauliflower mosaic virus $35 \mathrm{~S}$ promoter are either additive or synergistic, we observed a negative interaction in the PAL2 promoter, in which the -135 to -119 sequence specifically repressed a distant cis element. Parallel functional analysis of the promoter of a bean glycine-rich protein gene, which is specifically expressed in vascular tissues (Keller et al., 1989), has revealed the presence of a negative element that suppresses expression in cortical tissues (Keller and Baumgartner, 1991). As with the PAL2 negative cis element, this glycine-rich protein promoter element appears to have a dual function, in this case enhancing expression in vascular tissues in addition to suppressing cortical expression. Hence, combinatorial interactions involving the suppression of tissue-specific cis elements by modulatory cis elements with multiple, integrative functions may be a general feature of the functional architecture of promoters of genes involved in vascular development.

The presence of these negative regulatory elements and corresponding tissue-specific cryptic cis elements may provide a clue to the evolution of vascular promoters. Thus, the phloem conducting system appeared before xylem in the evolution of vascular plants, and in modern plants the inception of phloem differentiation occurs earlier than xylem differentiation (Raven et al., 1981). Hence, the presence of a cryptic phloem cis element might reflect the evolution of the PAL2 promoter from a primitive phloem promoter or nonspecific vascular promoter by introduction of an element that suppresses phloem expression and promotes xylem expression, associated with the evolution of an elaborate, lignified cell wall in xylem tracheary elements. In addition, this negative control system may provide a flexible mechanism for modifying tissue specificity under certain conditions. Although analysis of internal deletions showed that the cryptic phloem cis element does not affect tissue specificity during normal development, the $5^{\prime}$ deletions indicated that this region contains sequences that enhance the level of expression in xylem (Figure 1). Moreover, wounding of stem tissue induces PAL2 expression in the phloem and perivascular parenchymal cells (Liang et al., $1989 \mathrm{~b})$, and, hence, the phloem cis element may be activated by external stimuli, possibly through modulation of the negative regulatory element. In the case of wounding, this would alter tissue-specific expression for the synthesis of protective phenylpropanoid products in the tissues surrounding the vasculature (Liang et al., 1989b). Experiments are in progress to define the specific sequences involved in phloem expression in the mutated promoter and to delineate the function and regulation of this element in the intact promoter during development and in response to environmental changes.

The tissue specificities of the PAL2 promoter and mutated versions are maintained in stems, roots, and petioles even though the xylem and phloem are organized in different patterns in these organs. In the case of deletion $\Delta 178-640$, this conservation extends to expression in the relatively undifferentiated parenchymal cells that surround the vasculature of the stem and petiole, as well as in the specialized parenchymal cells of the pericycle and endodermis that encase the vascular cylinder of the root. These observations suggest that the xylem and phloem cis elements we have identified respond to signal pathways cued by genes that determine vascular patterning. The delineation of several distinct cis-acting elements with clearly defined properties in the PAL2 promoter will allow the characterization of individual regulatory elements in different sequence contexts, as well as the search for trans-acting factors that bind to these sequences. These studies provide the basis for elucidation of the molecular mechanisms governing vascular differentiation by biochemical analysis working back-stepwise from the PAL2 trans-acting factors and also by genetic approaches in which transgenic Arabidopsis plants containing marker genes fused to the PAL2 promoter or synthetic tissue-specific promoters are used to screen for novel mutants in the underlying signal pathways.

\section{METHODS}

\section{Deletion Mutants}

The $5^{\prime}$ deletions were generated by exonuclease III/S1 nuclease digestion of the phenylalanine ammonia-lyase $2 / \beta$-glucuronidase (PAL2-GUS) gene fusion pBP61(1) (Liang et al., 1989b). To generate internal deletions, $5^{\prime}$ and $3^{\prime}$ deletions were first obtained independently by the same method using the Dral restriction fragment of the PAL2 promoter (-991 to +53 ) for the $3^{\prime}$ deletions (Cramer et al., 1989) and the EcoRl fragment from PBPG1, which contains the PAL2-GUS gene fusion cloned in PGEM7 (Liang et al., 1989b), for the $5^{\prime}$ deletions. Appropriate combinations of these $5^{\prime}$ and $3^{\prime}$ deletions were ligated using the Aatll site of the pGEM7 polylinker and cloned into the EcoRI-HindIII site of the binary vector pBIN19 (Bevan, 1984). The constructs were confirmed by direct double-stranded nucleotide sequencing with the appropriate primers (Sambrook et al., 1989). 


\section{Transformation}

The binary vectors were transferred from Escherichia coli into Agrobacterium tumefaciens LBA4404 by the direct transformation method. Agrobacterium-mediated tobacco leaf disc transformation and regeneration of transgenic plants were performed as previously described (Rogers et al., 1986). Putative transformants displaying kanamycin resistance were confirmed by analysis of DNA gel blots (Southern, 1975) of genomic DNA probed with PAL2 promoter and GUS coding sequences. Transiormants were grown at $25^{\circ} \mathrm{C}$ under a 16 -hr light ( 115 $\mu \mathrm{E}) / 8$-hr dark cycle.

\section{GUS Assays}

Fluorometric assay of GUS activity in tissue extracts and histochemical staining of GUS activity in situ were performed as previously described (Liang et al., 1989b), except that root sections were embedded in $4 \%$ agarose prior to hand sectioning.

\section{DNA Blots}

DNA was isolated from tobacco leaves by the cetyltrimethylammonium bromide procedure (Murray and Thompson, 1980). DNA gel blots of genomic DNA from transgenic plants were probed with the 2.2-kb HindIII-EcoRI fragment from pB/101 that contains the GUS gene (Jefferson et al., 1987) or the 0.2-kb Rsal-Haelll fragment from the PAL2 promoter using standard procedures (Sambrook et al., 1989). The probes were labeled by random priming to a specific activity of 1 to $5 \times 10^{9} \mathrm{cpm} / \mu \mathrm{g}$ DNA.

\section{ACKNOWLEDGMENTS}

We thank Cindy Doane for assembling the manuscript and Armand Seguin for scientific discussions and help with the artwork. This research was supported by grants to C.J.L. from the Samuel Roberts Noble Foundation and the Hermann Frasch Foundation. A.L. and J.P.T. thank the Spanish Ministry of Science and Education and the Fulbright Foundation, respectively, for research fellowships.

Received November 7, 1991; accepted January 24, 1992.

\section{REFERENCES}

Benfey, P.N., and Chua, N.-H. (1990). The cauliflower mosaic virus $35 \mathrm{~S}$ promoter: Combinatorial regulation of transcription in plants. Science 250, 959-966

Bevan, M. (1984). Binary Agrobacterium vectors for plant transformation. Nucl. Acids Res. 12, 8711-8721.

Bevan, M., Shufflebottom, D., Edwards, K., Jefferson, R., and Schuch, W. (1989). Cell- and tissue-specific expression of a phenylalanine ammonia-lyase promoter in transgenic plants. EMBO J. 8, 1899-1906.
Bolwell, G. P., Bell, J.N., Cramer, C.L., Schuch, W., Lamb, C.J., and Dixon, R.A. (1985). L-Phenylalanine ammonia-lyase from Phaseolus vulgaris: Characterization and differential induction of multiple forms from elicitor-treated cell suspension cultures. Eur. J. Biochem. 149, 411-419.

Cramer, C.L., Edwards, K., Dron, M., Liang, X., Dildine, S.L., Bolwell, G.P., Dixon, R.A., Lamb, C.J., and Schuch, W. (1989). Phenylalanine ammonia-lyase gene organization and structure. Plant Mol. Biol. 12, 367-383.

Elkind, Y., Edwards, R., Mavandad, M., Hedrick, S.A., Ribak, O., Dixon, R.A., and Lamb, C.J. (1990). Abnormal plant development and down-regulation of phenylpropanoid biosynthesis in transgenic tobacco containing a heterologous phenylalanine ammonia-lyase gene. Proc. Natl. Acad. Sci. USA 87, 9057-9061.

Haddon, L.E., and Northcote, D.H. (1975). Quantitative measurement of the course of bean callus differentiation. J. Cell Sci. 17, 11-26.

Jefferson, R.A., Kavanagh, T.A., and Bevan, M.W. (1987). GUS fusions: $\beta$-Glucuronidase as a sensitive and versatile gene fusion marker in higher plants. EMBO J. 6, 3901-3907.

Keller, B., and Baumgartner, C. (1991). Vascular-specific expression of the bean GRP 1.8 gene is negatively regulated. Plant Cell 3 , 1051-1061.

Keller, B., Schmid, J., and Lamb, C.J. (1989). Vascular expression of a bean cell wall glycine-rich protein- $\beta$-glucuronidase gene fusion in transgenic tobacco. EMBO J. 8, 1309-1314.

Lamb, C.J., Lawton, M.A., Dron, M., and Dixon, R.A. (1989). Signals and transduction mechanisms for activation of plant defense mechanisms. Cell 56, 215-224.

Liang, X., Dron, M., Cramer, C.L., Dixon, R.A., and Lamb, C.J. (1989a). Differential regulation of phenylalanine ammonia-lyase genes during plant development and by environmental cues. J. Biol. Chem. 264, 14486-14492.

Liang, X., Dron, M., Schmid, J., Dixon, R.A., and Lamb, C.J. (1989b). Developmental and environmental regulation of a phenylalanine ammonia-lyase- $\beta$-glucuronidase gene fusion in transgenic tobacco plants. Proc. Natl. Acad. Sci. USA 86, 9284-9288.

Lois, R., Dietrich, A., Hahlbrock, K., and Schulz, W. (1989). A phenylalanine ammonia-lyase gene from parsley: Structure, regulation and identification of elicitor-and light-responsive cis-elements. EMBO J. 8, 1641-1648.

Lynn, D.G., and Chang, M. (1990). Phenolic signals in cohabitation: Implications for plant development. Annu. Rev. Plant Physiol. Plant Mol. Biol. 41, 497-526.

Miesfeld, R., Godowski, P.J., Maler, B.A., and Yamamoto, K.R. (1987). Glucocorticoid receptor mutants that define a small region sufficient for enhanced activation. Science 236, 423-426.

Minami, E.I., Ozeki, Y., Matsuoka, M., Koizuka, N., and Tanaka, T. (1989). Structure and some characterization of the gene for phenylalanine ammonia-lyase from rice plants. Eur. J. Biochem. 185, 19-25.

Murray, M.G., and Thompson, W.F. (1980). Rapid isolation of high molecular weight DNA. Nucl. Acids Res. 8, 4321-4325.

Ohl, S., Hedrick, S.A., Chory, J., and Lamb, C.J. (1990). Functional properties of a phenylalanine ammonia-lyase promoter from Arabidopsis. Plant Cell 2, 837-848.

Raven, P.H., Evert, R.F., and Curtis, H. (1981). Biology of Plants, 3rd ed. (New York: Worth). 
Rogers, S.G., Horsch, R.B., and Fraley, R.T. (1986). Gene transfer in plants: Production of transformed plants using Ti plasmid vectors. Methods Enzymol. 118, 627-640.

Rubery, P.H., and Northcote, D.H. (1968). Site of phenylalanine ammonia-lyase activity and the synthesis of lignin during xylem differentiation. Nature 219, 1230-1232.

Sakal, D.D., Helms, S., Carlstedt-Duke, J., Gustaffson, J.A., Rottman, F.M., and Yamamoto, K.R. (1988). Hormone-mediated repression: A negative glucocorticoid response element from the bovine prolactin gene. Genes Dev. 2, 1144-1154.

Sambrook, J., Fritsch, E.F., and Maniatis, T. (1989). Molecular Cloning: A Laboratory Manual, 2nd ed. (Cold Spring Harbor, NY: Cold Spring Harbor Laboratory).

Southern, E.M. (1975). Detection of specific sequences among DNA fragments separated by gel electrophoresis. J. Mol. Biol. 98, 503-517. 
cis-element combinations determine phenylalanine ammonia-lyase gene tissue-specific expression patterns.

A Leyva, X Liang, J A Pintor-Toro, R A Dixon and C J Lamb

Plant Cell 1992;4;263-271

DOI 10.1105/tpc.4.3.263

This information is current as of January 21, 2013

Permissions

eTOCs

CiteTrack Alerts

Subscription Information
https://www.copyright.com/ccc/openurl.do?sid=pd_hw1532298X\&issn=1532298X\&WT.mc_id=pd_hw15322 $98 \mathrm{X}$

Sign up for eTOCs at:

http://www.plantcell.org/cgi/alerts/ctmain

Sign up for CiteTrack Alerts at:

http://www.plantcell.org/cgi/alerts/ctmain

Subscription Information for The Plant Cell and Plant Physiology is available at: http://www.aspb.org/publications/subscriptions.cfm 